\title{
Type II Diabetes Mellitus Prolongs Rocuronium Induced Neuromuscular Blockade Irrespective of Glycaemic Control
}

\author{
Aysun ŞAHIN 1 , [MD] \\ ORCID: 0000-0002-7883-3227 \\ Aysun ANKAY YILBAŞ 1,*,[MD] 0 \\ ORCID: 000-0001-6196-3191 \\ A. Gülsün PAMUK ', [MD] \\ ORCID: 0000-0001-8350-1270 \\ Başak AKÇA ${ }^{1}$, [MD] \\ ORCID: 0000-0003-4069-2462 \\ Filiz ÜZÜMCÜĞiL ', [MD] \\ ORCID: 0000-0001-9161-3248 \\ Nalan ÇELEBI ${ }^{1}$,[MD] \\ ORCID: 0000-0001-7472-6275 \\ 1 Hacettepe University Faculty of Medicine Department \\ of Anesthesiology, \\ * Corresponding Author: Başak Akça \\ Address: Hacettepe University Faculty of Medicine \\ Department of Anesthesiology, \\ Sihhiye, Ankara, 06100, TURKEY \\ Phone: +903123051255 \\ Fax: +903123109600 \\ e-mail:drbasakakca@gmail.com \\ DOI:10.32552/2019.ActaMedica.343
}

\section{nel ABSTRACT Cem}

Objective: Recovery from neuromuscular blockade could be delayed and the risk of postoperative residual neuromuscular blockade could be an important anesthetic consideration in diabetic patients undergoing general anesthesia due to decreased nerve conduction velocity. The aim of this study was to analyse the recovery from rocuronium and the risk of postoperative residual neuromuscular blockade in type-II diabetic patients undergoing general anesthesia with sevoflurane.

Materials and Methods: The patients undergoing general anesthesia with sevoflurane and rocuronium for abdominal surgery were included in this prospective observational study between December 2014 - May 2015. After rocuronium injection; time to reappearance of T1, T2, T3 and T4 and time to reaching train of four ratios of 0.7 and 0.9 were recorded. For detecting residual neuromuscular blockade, train of four ratios were recorded in the postoperative care unit at $5^{\text {th }}$ and $10^{\text {th }}$ minutes of arrival. The correlation between glycemic control and the risk of residual neuromuscular blockade was also evaluated via glycosylated haemoglobin (HbA1c) levels.

Results: A total of 82 patients (Group diabetes mellitus: 48 patients, Group non-diabetes mellitus: 34 patients) were included. The groups were comparable according to demographic data and other characteristics that can effect neuromuscular blockade. Times to reappearance of T1, T2, T3, T4 and to reaching a train of four ratio of 0.9 were significantly longer in Group diabetes mellitus. Although there was no difference between the train of four ratios at the arrival to the postoperative care unit, train of four ratios of Group diabetes mellitus were significantly lower at $5^{\text {th }}$ and $10^{\text {th }} \mathrm{min}$ utes at postoperative care unit. Poor glycaemic control was found not to affect the risk of residual neuromuscular blockade.

Conclusion: Even in the absence of known neurologic complications; the neuromuscular blockade effect of rocuronium is longer and the risk of residual neuromuscular blockade is higher in type-II diabetic patients irrespective of the degree of glycaemic control.

Keywords: Diabetes mellitus, residual neuromuscular blockade, Train-of-four

Received:04 March 2019, Accepted: 15 March 2019,

Published online: 30 March 2019

\section{INTRODUCTION}

Diabetes mellitus (DM) can lead to decreased nerve conduction velocity as a result of segmental demyelination and partial degeneration of nerve fibers and loss of motor units [1]. In vitro studies have shown that DM affects both metabolic and transmission functions at the neuromuscular junction due to impaired glucose transport and secretion of acetylcholine from the presynaptic motor nerve endings [2]. In addition, skeletal muscle damages such as muscular infarction and atrophy have been reported in diabetic patients $[3,4]$. Thus, recovery from neuromuscular blockade could be delayed and the risk of postoperative residual neuromuscular blockade could be an important anesthetic consideration in diabetic patients undergoing general anesthesia. Nitahara et al. showed that neuromuscular function recovery following vecuronium is prolonged in diabetic patients compared to non-diabetics, even in the absence of diabetic neuropathy or nephropathy [5]. However, the studies conducted with rocuronium, which has a more rapid onset of action and recovery profile, reveal controversial results. While 
Alper et al. [1] found rocuronium induced neuromuscular block not affected in type II DM during general anesthesia with isoflurane, Armendariz-Buil et al. [6] found increased risk of residual neuromuscular blockade with rocuronium in diabetics similar to the literature regarding vecuronium. Therefore, the aim of this study was to analyse the recovery from rocuronium and the risk of postoperative residual neuromuscular blockade in type II diabetic patients undergoing general anesthesia with sevoflurane.

\section{MATERIALS and METHODS}

The patients undergoing general anesthesia with sevoflurane and rocuronium for abdominal surgery were included in this prospective, observational, cross-sectional study design, in the time period between December 2014 and May 2015. The study was conducted in Hacettepe University Faculty of Medicine, Department of Anesthesiology and Reanimation after obtaining local ethical committee approval (17.12.2014/17, approval number: 16969557-1265). Written informed consent was obtained from all patients.

Patients with allergy to rocuronium, with neuromuscular diseases like Guillain-Barre syndrome, Duchenne muscular dystrophy and Myasthenia gravis, with type I diabetes mellitus, with a BMI $\nabla 18.5$ or $\bigotimes 30 \mathrm{~kg} / \mathrm{m}^{2}$, with American Society of Anesthesiology (ASA) physical status $>$ III, with any known neuropathy or nephropathy (due to DM or not), with hepatic or renal failure, patients receiving anticonvulsants or any other medications that can affect the response to neuromuscular blockers and patients aged $<18$ years were excluded from the study. Other exclusion criteria were having serum creatinine levels $>1.5 \mathrm{mg} / \mathrm{dL}$, serum glutamic-oxaloacetic transaminase (SGOT) and serum glutamic-pyruvic transaminase (SGPT) >45 IU and abnormal plasma concentrations of calcium, magnesium, sodium and potassium.

Following standard monitoring with electrocardiogram, non-invasive blood pressure (NIBP), pulse oximeter (SpO2), capnograph and bispectral index (BIS); preparations for neuromuscular monitoring was done by placing the two stimulating electrodes on the ulnar nerve at the wrist and two recording electrodes over the adductor pollicis muscle after cleaning the skin with alcohol to decrease resistance. The hand and forearm were fastened to the armrest to avoid interference. The neuromuscular transmission (NMT) module integrated in Avance CS2 Pro anesthesia machine (GE Healthcare Systems, United States) was used for neuromuscular monitoring. Anesthesia induction was performed with intravenous $2.5 \mathrm{mg} / \mathrm{kg}$ propofol and $1 \mathrm{mcg} / \mathrm{kg}$ fentanyl in all patients. After loss of the eyelid reflex, autocalibration was done and train of four (TOF) stimuli were applied with the system automatically identifying supramaximal stimulating currents. After the supramaximal stimulating current was recorded, rocuronium $0.6 \mathrm{mg} / \mathrm{kg}$ iv was given to the patients. TOF stimuli was applied every $15 \mathrm{sec}-$ onds with a duration of $0.2 \mathrm{msec}$ and a frequency of $2 \mathrm{~Hz}$. When the TOF ratio became 0 , endotracheal intubation was performed. Anesthesia was maintained with $2 \%$ sevoflurane in a mixture of $50 \%$ oxygen and $50 \%$ air. Intraoperative analgesia was maintained with remifentanil infusion $(0.1-0.5 \mathrm{mcg} / \mathrm{kg} /$ min). End-tidal $\mathrm{CO} 2$ values were measured continuously and ventilation was controlled to maintain normocapnia (30-35 mmHg). Core temperature via a nasopharyngeal probe was also monitored during the intraoperative period. When return of T2 was seen, an additional dose of rocuronium $(0.15 \mathrm{mg} / \mathrm{kg}$ iv) was administered. In case of an increase of heart rate or blood pressure $\geq 15 \%$ above the baseline, 50 mcg bolus fentanyl was administered intravenously. Times from rocuronium administration to the return of first (T1), second (T2), third (T3) and fourth (T4) TOF responses, and times to reach TOF ratio of 0.7 and 0.9 were recorded. Total duration of anesthesia and surgery, reverse-extubation time, time to extubation and time to eye opening were noted. Duration of anesthesia was defined as time from anesthesia induction to the cessation of sevoflurane. Duration of surgery was defined as time from the first surgical incision to the last skin suture. Times to extubation and eye opening were defined as the times from cessation of sevoflurane to the extubation of patient's trachea and spontaneous opening of eyes respectively. Reverse-extubation time was defined as the time between administration of reversal agent for neuromuscular blockade and extubation. The other parameters recorded were age, sex, weight, body mass index, ASA classification, coexisting diseases, drug history, and laboratory tests including blood urea nitrogen (BUN), creatinine, $\mathrm{HbA1c}$ and fasting blood glucose levels.

All patients received $0.1 \mathrm{mg} / \mathrm{kg}$ iv morphine (calculated according to the ideal body weight) and 1 gr paracetamol for postoperative analgesia, 20-25 minutes before the end of the surgery as a standard hospital policy. At the end of the surgery, if the TOF ratio was $<0.9$, neostigmine $(0.04 \mathrm{mg} / \mathrm{kg}$ combined with $0.01 \mathrm{mg} / \mathrm{kg}$ atropine iv) or sugammadex ( $2 \mathrm{mg} /$ $\mathrm{kg}$ iv) was administered for antagonizing the neuromuscular blockade. As both drugs were commonly used in our clinic's standard practice and the study 
design was observational, the study group did not interfere to the choice of the anesthesiologist responsible for patient care. The type of the antagonizing drug used, simultaneous TOF ratio and the time between administration of antagonizing drug and extubation were recorded. All patients were extubated when TOF ratio became 0.9. The patients were then transferred to post-anesthesia care unit (PACU) and TOF ratios were recorded in PACU at the 5th and 10th minutes of arrival in addition to the standard monitoring, including electrocardiogram, non-invasive blood pressure, pulse oximeter and peripheral skin temperature. Since the patients were awake, they were warned of a brief discomfort that would accompany TOF stimulus and asked again for oral consent before TOF measurements were performed. All patients scheduled for abdominal surgery were actively warmed with BairHugger blanket system covering the extremities at $38^{\circ} \mathrm{C}$ and were covered with warm blankets at arrival to PACU as a standard procedure of our clinic. Discharge from PACU to the wards was accomplished when the Alderete score became at least 9 according to the usual hospital policy. The duration of stay at PACU and time to the Aldrete score reaching 9 were recorded.

\section{Statistical Analysis}

Data analysis was performed by using IBM SPSS version 20.0 software (IBM Corporation, Armonk, NY,
USA). To compare the data in two groups, student t-test and Mann-Whitney $U$ test were used for continuous variables, where applicable. Categoric variables were compared using chi-square test. The values that were normally distributed were presented as means \pm standard deviation. Ordinal data were expressed as median (min-max).

A p value $<0.05$ was considered significant.

\section{RESULTS}

A total of 82 patients (Group DM: 48 patients, Group non-DM: 34 patients) were included between December 2014 and May 2015. The groups were comparable according to demographic data and other characteristics that can affect neuromuscular blockade (Table 1). ASA status was significantly different between groups, because all the patients in Group DM were classified as at least ASA ॥ even when they did not have any other coexisting disease. Vital parameters (non-invasive blood pressure, $\mathrm{SpO} 2$, heart rate) and body temperature measured just before induction of anesthesia were also comparable between groups. Most commonly performed surgery was laparoscopic cholecystectomy in both groups. Hypertension, chronic obstructive pulmonary disease and coronary artery disease were the most common comorbidities in both groups.

Table 1. Demographic data and preoperative laboratory parameters [Normally distributed data were expressed as mean \pm standard deviation, non-normally distributed data were expressed as median (minimum-maximum)

\begin{tabular}{|l|c|c|c|}
\hline & Group DM (n=48) & Group NDM (n=34) & p \\
\hline Sex (F/M) (n) & $22 / 26$ & $14 / 20$ & 0.424 \\
\hline Age (year) & $62.45 \pm 9.09$ & $58.64 \pm 8.71$ & 0.175 \\
\hline BMI (kg/m) & $26.6(20.0-45.8)$ & $26.1(20.3-29.8)$ & $0.000^{*}$ \\
\hline ASA (I/II/III) & $0 / 36 / 12$ & $13 / 20 / 1$ & 0.826 \\
\hline BUN (mg/dL) & $13.8 \pm 3.2$ & $14.0 \pm 3.9$ & 0.659 \\
\hline Creatinine (mg/dL) & $0.7 \pm 0.1$ & $99.7 \pm 11.9$ & 0.066 \\
\hline $\begin{array}{l}\text { Preoperative blood glu- } \\
\text { cose (g/dL) }\end{array}$ & $109.4 \pm 21.2$ & $110.64 \pm 46.23$ & 0.059 \\
\hline $\begin{array}{l}\text { Duration of anesthesia } \\
\text { (min) }\end{array}$ & $129.47 \pm 42.15$ & $97.64 \pm 45.18$ & 0.083 \\
\hline Duration of surgery (min) & $114.27 \pm 40.09$ & 0.7 & \\
\hline
\end{tabular}

* $p<0.005$ F/M: female/male, BMI: body mass index, ASA: American Society of Anesthesiologists, BUN: blood urea nitrogen 
All patients were intubated when TOF ratio became zero and an additional dose of rocuronium was administered when the return of $\mathrm{T} 2$ was seen. Times to reappearance of $\mathrm{T} 1, \mathrm{~T} 2, \mathrm{~T} 3, \mathrm{~T} 4$ and to reaching a TOF ratio of 0.9 were statistically significantly longer in Group DM (Table 2).

Table 2. Time to appearance of $\mathrm{T} 1, \mathrm{~T} 2, \mathrm{~T} 3, \mathrm{~T} 4$ and to reaching $\mathrm{TOF}$ ratios of 0.7 and 0.9 [Normally distributed data were expressed as mean \pm standard deviation, non-normally distributed data were expressed as median (minimum-maximum)

\begin{tabular}{|l|c|c|c|}
\hline Time (min) & Group DM $(\mathrm{n}=48)$ & Group NDM $(\mathrm{n}=34)$ & $\mathrm{p}$ \\
\hline T1 & $42(15-160)$ & $35(15-90)$ & $0.007^{*}$ \\
\hline T2 & $60(20-210)$ & $45(30-130)$ & $0.001^{*}$ \\
\hline T3 & $76.5(30-210)$ & $55(35-190)$ & $0.008^{*}$ \\
\hline T4 & $87.5(45-220)$ & $63.5(40-200)$ & $0.016^{*}$ \\
\hline TOF 0.7 & $117.5 \pm 48$ & $102.7 \pm 47.8$ & 0.173 \\
\hline TOF 0.9 & $138(50-240)$ & $86(55-220)$ & $0.017^{*}$ \\
\hline
\end{tabular}

${ }^{*} p<0.005$

The time between administration of rocuronium and intubation was significantly longer in Group DM (Group DM: 3.5 (1.0-3.0) min, Group NDM: 2 (1.0-4.0) min, $p<0.001)$. But there was no difference in the time between first rocuronium dose and the need for an additional dose (Group DM: 70 (20-175) min, Group NDM: 62.5 (35-150.4), $p=0.873$ ). As the study design was prospective observational, the study group did not interfere with the choice of using a reversal agent for neuromuscular blockade or the type of the drug. The neuromuscular blockade was antagonized in all patients and the antagonizing drugs used were neostigmine $(0.05 \mathrm{mg} / \mathrm{kg})$ and sugammadex $(2 \mathrm{mg} / \mathrm{kg})$. The time from the administration of the reversal agent to extubation (reverse-extubation time) was significantly longer in Group DM (11.3 $\pm 6.8 \mathrm{~min})$ compared to Group NDM $(6.2 \pm 3.5 \mathrm{~min})(p<0.001)$ but it did not differ according to the type of the reversal agent. Similarly, extubation and eye-opening times were significantly longer in Group DM compared to Group NDM $(p<0.001)$.

All patients were extubated when TOF ratio became 0.9 and then transferred to PACU but there was a significant decrease in TOF ratios measured at 5th and 10th minutes at PACU in Group DM compared to Group NDM. This difference was regardless of the type of the reversal agent (neostigmin/sugammadex) used to antagonize neuromuscular blockade (Table 3). There were 3 patients whose TOF ratio were $<0.8$ at PACU in Group DM, reversal agent used was neostigmine in 2 of them and sugammadex in 10 one of them. Only one patient in this group who received neostigmine revealed symptoms of respiratory difficulty with $\mathrm{SpO} 287 \%$ and he was treated with $2 \mathrm{mg} / \mathrm{kg}$ sugammadex. None of the patients in Group NDM showed a decrease of TOF ratio below 0.9 at PACU. Vital parameters other than $\mathrm{SpO} 2$ values were comparable in both groups at PACU. SpO2 values measured at 5th min of stay at PACU were lower in Group DM compared to Group NDM (Group DM: 96(87-99), Group NDM: 98(90-100), $p=0.012$ ). The duration of stay at PACU was significantly longer in Group DM (Group DM: 25 (15-35) min, Group NDM: 20 (15-30), $p<0.001)$. Aldrete scores of patients in Group DM were also lower compared to Group NDM at arrival, 5th and 10th min $(p<0.001)$ but the difference disappeared later and all patients were discharged from PACU with a minimum Aldrete score of 9.

Vital parameters (SpO2, heart rate, NIBP, etCO2, BIS) were comparable in both groups during the operation. Although there were no differences in core body temperature before and after induction of anesthesia and the operating room temperatures (21$23^{\circ} \mathrm{C}$ ) and warming procedures were standardized for all patients, we saw a tendency to hypothermia in Group DM compared to Group NDM. After the 30th min of intraoperative period, the body temperature of Group DM became significantly lower compared to Group NDM (at 30th min, Group DM: 36.0 (35.0-36.5) ${ }^{\circ} \mathrm{C}$, Group NDM: 36.0 (35.6-36.5) ${ }^{\circ} \mathrm{C}$, $\mathrm{p}=0.026$; at 60th min, Group DM: $36.0(35.0-36.0)^{\circ} \mathrm{C}$, Group NDM: 36.0 (35.6-36.0) $\left.{ }^{\circ} \mathrm{C}, \mathrm{p}=0.002\right)$. The core 
Table 3. TOF ratios at post-anesthesia care unit (PACU) [Data were expressed as median (minimum-maximum)

\begin{tabular}{|c|c|c|c|}
\hline \multicolumn{4}{|c|}{ Neostigmin for reversal of NMB $(n=52)$} \\
\hline & Group DM (n=31) & Group NDM $(n=21)$ & $\mathrm{p}$ \\
\hline TOF at $5^{\text {th }} \min$ & $0.91(0.59-1)$ & $0.94(0.9-1)$ & $0.001^{*}$ \\
\hline TOF at $10^{\text {th }} \min$ & $0.91(0.7-1)$ & $0.96(0.92-1)$ & $0.000^{*}$ \\
\hline \multicolumn{4}{|c|}{ Sugammadex for reversal of NMB $(n=30)$} \\
\hline & Group DM $(n=17)$ & Group NDM $(n=13)$ & $\mathrm{p}$ \\
\hline TOF at $5^{\text {th }} \min$ & $0.89(0.7-1)$ & $0.92(0.9-1)$ & $0.043^{*}$ \\
\hline TOF at $10^{\text {th }} \min$ & $0.92(0.87-1)$ & $0.94(0.92-1)$ & 0.183 \\
\hline \multicolumn{4}{|c|}{ Regardless of reversal agent $(n=82)$} \\
\hline & Group DM $(n=48)$ & Group NDM (n=34) & $p$ \\
\hline TOF at $5^{\text {th }} \min$ & $0.91(0.59-1)$ & $0.94(0.9-1)$ & $0.001^{*}$ \\
\hline TOF at $10^{\text {th }} \mathrm{min}$ & $0.92(0.7-1)$ & $0.96(0.92-1)$ & $0.001^{*}$ \\
\hline
\end{tabular}

${ }^{*} p<0.005$

temperatures measured at the 90th and 120th min were similar in both groups. Although median values at the arrival time at PACU seemed similar at first look, there were more hypothermic patients in Group DM and the difference was found statistically significant (Group DM: 36.0 (35.5-36.5) ${ }^{\circ} \mathrm{C}$, Group NDM: 36.0 (35.7-37.0) ${ }^{\circ} \mathrm{C}, \mathrm{p}=0.018$ ).

We did not see any intraoperative complications other than a slight hypotension (systolic blood pressure $<80 \mathrm{mmHg}$ ) that occurred in 3 patients. One was a non-diabetic patient and the hypotension, which was due to acute surgical bleeding, was treated with crystalloids and erythrocyte suspension infusion. Hypotension that occurred in the 2 diabetic patients was treated with crystalloid infusion and an ephedrine bolus ( $5 \mathrm{mg}$ ); this complication was thought to be related to a possible autonomic neuropathy.

None of our patients had known neuropathic or nephropathic complications due to DM. The time from the diagnosis of DM was $\leq 5$ years in $45.8 \%$ and $\leq 10$ years in $83.3 \%$ of diabetic patients. Mean $\mathrm{HbA1c}$ level was $6.82 \pm 1.45 \%$ in Group DM and $23 \%$ of patients were on routine insulin treatment for their diabetes. Poor glycaemic control was found not to affect the risk of residual neuromuscular blockade (RNMB).

\section{DISCUSSION}

This study revealed that times to reappearance of $\mathrm{T} 1, \mathrm{~T} 2, \mathrm{~T} 3, \mathrm{~T} 4$ and to reaching a TOF ratio of 0.9 were significantly longer in diabetic patients compared to the control group. Besides this, TOF ratios of diabetic patients showed a significant decrease at the 5th and 10th minutes of PACU arrival regardless of the type of the reversal agent. Poor glycemic control did not seem to affect the risk of RNMB.

The underlying mechanisms of prolonged neuromuscular blockade in diabetic patients are not only axon loss, demyelination and partial degeneration in the motor nerve endings, but also atrophy and infarction of skeletal muscles [1-4]. The reduction in single twitch amplitude and the return of $\mathrm{T} 1, \mathrm{~T} 2, \mathrm{~T} 3$ and $\mathrm{T} 4$ are related to the degree of neuromuscular block at the postsynaptic muscle membrane; however, TOF ratio shows the degree of block at presynaptic region due to the inhibition of presynaptic subtype of nicotinic acetylcholine receptors $[7,8]$. Since all the parameters were found longer in diabetic patients in this study, both pre- and postsynaptic regions can be thought to be affected in diabetic patients.

Recovery of neuromuscular function after vecuronium has been shown to be prolonged in type II DM patients [5]. Rocuronium is thought to be a safer agent with its rapid onset and recovery profile and inactive metabolites. Alper et al. found no alterations in rocuronium induced neuromuscular blockade in patients with type $2 \mathrm{DM}$ (with a median period of 8.5 years since diagnosis) during general anesthesia 
with isoflurane [1]. However, Armendariz-Buil et al. 's [6] study conducted under sevoflurane anesthesia suggests increased risk of RNMB after rocuronium similar to our results. The effect of inhalational anesthetics, especially sevoflurane, on neuromuscular junction to delay the neuromuscular block can be one of the most important factors leading to these controversial results. Another condition affecting the differences between studies can be the mean age of patients. The mean age of patients in the current study and Armendariz-Buil et al's [6] study seem to be older than Alper et al's [1] study that found no difference in neuromuscular blockade between diabetic and non-diabetic groups.

Poor glycemic control is generally associated with increased risk of neuropathy in diabetic patients. A cross-sectional study also revealed that longer duration of diabetes ( $>6$ years) and poor glycemic control ( $\mathrm{HbA1c}>8.0 \%)$ were associated with poorer muscle quality even in apparently healthy subjects [9]. Contrary to the expectations, Armendariz-Buil et al. [6] couldn't find a correlation between poor glycemic control and increased risk of RNMB with rocuronium. Our results were also consistent with that study. However, relatively small sample sizes of both studies should be considered. Additionally, peripheral neuropathy due to DM actually develops within years but $\mathrm{HbA1c}$ reflects only the glycemic control in the last 4-6 weeks.

The onset time of rocuronium was also found significantly longer in diabetic patients in the current study. Although the onset time of vecuronium has been found similar in diabetics and non-diabetics in few clinical and experimental studies [10], the data in the literature related to onset time of neuromuscular blockers could be considered limited. As two groups were comparable according to demographic and clinical parameters like BMI, hepatic or renal failure, the use of other medications which may include the pharmacodynamics and pharmacokinetics of anesthetic drugs, this delay in the onset time of rocuronium in diabetic patients in the current study might not be attributed to these factors. In fact, as the motor neuron is impaired, we can expect that the onset time of neuromuscular blockade could be earlier in diabetic patients. A decrease in cardiac output and left ventricular dysfunction in diabetic patients had been thought to offset the sensitivity to vecuronium in the previous studies that found no difference in the onset time [11]. Hypertension $(n=20$ in Group DM) and coronary artery disease ( $n=19$ in Group DM) were the most commonly seen comorbidities in our study. Although we did not measure cardiac output preoperatively, the delay in onset time of rocuronium in our study may be due to a possible decrease in cardiac output. Additionally, there may be possible problems regarding neuromuscular monitoring in diabetic patients. For example, there are two reported diabetic cases who were diagnosed as polyneuropathy postoperatively after having problems with intraoperative neuromuscular monitoring via ulnar nerve [12]. None of the patients in the present study had been diagnosed with neuropathy prior to surgery and, in keeping with the standard operating practices at our hospital, no additional tests were conducted to discover cases of undiagnosed neuropathy.

\section{CONCLUSION}

We have found the time from the administration of rocuronium to intubation, the time from the administration of the reversal agent to extubation, and the time from extubation to eye opening to all be significantly longer for patients with type II diabetes mellitus. The risk of RNMB was higher in type II diabetic patients irrespective of the degree of glycaemic control. It is therefore recommended that patients with DM be admitted to surgery under strict TOF monitorization even in the absence of known neuropathy, and the dosage of all medication in these cases be regulated accordingly.

\section{CONFLICT OF INTEREST}

\section{None}




\section{ne) REFERENCES Cem}

[1] Alper I, Ulukaya S, Makay O, et al. The pharmacodynamic effects of rocuronium during general anesthesia in patients with type 2 diabetes mellitus. Minerva Anestesiol 2010; 76: 115-19.

[2] Constantini S, Schiller $Y$, Cohen AM, et al. Pathophysiology of the neuromuscular junction in diabetic rats. Isr J Med Sci 1987; 23: 101-06.

[3] Rocca PV, Alloway JA, Nashel DJ. Diabetic muscular infarction. Semin Arthritis Rheum 1993; 22: 280-87.

[4] Van Slyke MA, Ostrov BE. MRI evaluation of diabetic muscle infarction. Magn Reson Imaging 1995; 13: 325-29.

[5] Nitahara K, Sugi $Y$, Shigematsu K, et al. Recovery of trainof-four ratio to 0.70 and 0.90 is delayed in type 2 diabetes with vecuronium-induced neuromuscular block. Eur J Anaesthesiol 2013; 30: 80-84.

[6] Armendáriz-Buil I, Lobato-Solores F, Aguilera-Celorrio L, et al. Residual neuromuscular block in type II diabetes mellitus after rocuronium: a prospective observational study. Eur J Anaesthesiol 2014; 31: 411-16.
[7] Fagerlund MJ, Eriksson LI. Current concepts in neuromuscular transmission. Br J Anaesth 2009; 103: 108-14.

[8] Fuchs-Buder T, Claudius C, Skovgaard LT, et al. Good clinical research practice in pharmacodynamic studies of neuromuscular blocking agents II: the Stockholm revision. Acta Anaesthesiol Scand 2007; 51: 789-808.

[9] Park SW, Goodpaster BH, Strotmeyer ES, et al. Decreased muscle strength and quality in older adults with type 2 diabetes: the health, aging, and body composition study. Diabetes 2006; 55: 1813-18.

[10] Clark L, Leece EA, Brearley JC. Diabetes mellitus affects the duration of action of vecuronium in dogs. Vet Anaesth Analg 2012; 39: 472-79.

[11] Nesto RW, Zarich SW, Jacoby RM, et al. Heart disease in diabetes. In: Kahn CR, Weir GC, (eds). Joslin's Diabetes Mellitus. 13. Ed. Pennsylvania; Lea \& Febiger, 1994: 844-45.

[12] Knüttgen $D$, Bremerich $D$, Rings J, et al. Failure of relaxometry in diabetic polyneuropathy. Anesthesist 1992; 41: 559-63. 